\title{
Penning Trap at IGISOL
}

\author{
J. Szerypo ${ }^{\text {a }}$, A. Jokinen ${ }^{\text {a }}$, V. S. Kolhinen ${ }^{\mathrm{a}}$, A. Nieminen ${ }^{\mathrm{a}}$, \\ S. Rinta-Antila ${ }^{a}$ and J. Äystö ${ }^{b}$ \\ ${ }^{\mathrm{a} D e p a r t m e n t}$ of Physics, University of Jyväskylä, \\ P.O. Box 35 (Y5), FIN-40351 Jyväskylä, Finland \\ ${ }^{\mathrm{b}}$ CERN, CH-1211 Geneva 23, Switzerland
}

\begin{abstract}
The IGISOL facility at the Department of Physics of the University of Jyväskylä (JYFL) is delivering radioactive beams of short-lived exotic nuclei, in particular the neutron-rich isotopes from the fission reaction. These nuclei are studied with the nuclear spectroscopy methods. In order to substantially increase the quality and sensitivity of such studies the beam should undergo beam handling: cooling, bunching and isobaric purification. The first two processes are performed with the use of an RFQ cooler/buncher. The isobaric purification will be made by a Penning trap placed after the RF-cooler element. This contribution describes the current status of the Penning trap project and its future prospects. The latter comprise the precise nuclear mass measurements, nuclear spectroscopy in the Penning trap interior as well as the laser spectroscopy on the extracted beams.
\end{abstract}

A project for improving the quality of radioactive ion beams produced at IGISOL has been started in 1997 [1,2]. This improvement is achieved due to a radioactive beam handling, which consists of three steps: beam cooling, bunching and purification. The first two steps are done with a use of an RFQ cooler/buncher (see [3]). The beam purification is performed with the use of a cylindrical Penning trap similar to the one described in [4]. In this trap the ions are confined in three dimensions by a superposition of static quadrupole electric and homogeneous magnetic fields. The magnetic field confines the ions in two dimensions in a plane perpendicular to the field direction. A confinement in the third, magnetic field direction (parallel to the trap axis) is done by a quadrupole electric field. This field is created by a set of cylindrical (ring) electrodes in such a way that the central part of the electrode set is put to a negative potential, whereas the outer electrodes are at positive potential. This way an axial potential well for the ions with a minimum at the trap center is formed for positively charged species.

Preprint submitted to Elsevier Preprint 31 July 2000 
A mass-selective buffer gas cooling technique [5] will be used for purification. In order that the purification could take place, the trap interior has to be filled with a buffer gas at low pressure $\left(10^{-3}-10^{-4}\right.$ mbar $)$, usually helium. A lowenergy (of the order of $100 \mathrm{eV}$ ) ion beam coming from the RFQ cooler/buncher is captured in the trap center and then the buffer gas cooling can start. For this purpose, the central ring electrode is segmented azimuthally into 4 segments. The segments are supplied with an oscillating RF-potential so that an oscillating quadrupole field in the azimuthal plane is created. The RF-frequency is chosen so that it is equal to the cyclotron frequency of the ions of interest, which are usually mixed with other, contaminating ions. The joint action of the RF-field and the buffer gas is cooling and centering the ions of interest on the trap axis, whereas the contaminants not centered. The ions of interest are then ejected through a small hole in the endcap of the trap. This process can have a high mass resolving power, of the order of $10^{5}$, which permits to reject even isobaric contaminants [4]. This is particularly important for the experimental program at IGISOL, which in a future will be centered on the exotic neutron-rich nuclei, produced in a fission reaction. The isobaric purification will allow for rejecting of all unwanted members of the isobaric chain, leaving only a species of interest. This will significantly improve signal-to-background ratio, sensitivity and precision of the experiments, and will extend the range of the isotopes investigated.

The task of the purification Penning trap at IGISOL is to:

- accept cooled (continuous or bunched) beams from the RFQ cooler/buncher, - perform the isobaric purification,

- deliver clean, monoisotopic bunched beams for nuclear spectroscopy investigations, precise nuclear mass measurements and laser spectroscopy experiments.

In order to achieve this goal, it is planned to place the Penning trap inside a $\mathrm{B}=7 \mathrm{~T}$ superconducting magnet. This magnet was delivered already by Magnex Scientific Ltd. and installed at the IGISOL area in November 1999 (see Fig. 1). It is a solenoid with two homogeneous magnetic field regions (inhomogeneities within $1 \mathrm{~cm}^{3}$ volume below $10^{-6}$ and $10^{-7}$, respectively). The isobaric purification of radioactive beam will be performed in the cylindrical Penning trap positioned in the first region. Completing the purification trap will make the first, very important step in the Penning trap development at IGISOL and will open new possibilities for collinear laser and nuclear spectroscopy experiments. The former will profit already of the presence of the RFQ cooler/buncher, delivering a bunched beam with very good emittance [3]. Isobaric purity will still add a lot to the sensitivity of these measurements. The nuclear specroscopy set-up will utilize a variety of gamma, X-ray and particle detectors. The bunched monoisotopic beam from the trap will be transferred to the measurement position and implanted in a collecting foil or a movable tape surrounded by the above-mentioned detector set-up. In this 
set-up, significant increase in the measurement sensitivity and precision is expected as compared to the standard (no-trap) situation, which was already mentioned before.

The second step of the Penning trap development will consist of building a second Penning trap for the precise nuclear mass measurements of radioactive ions. It will be placed directly after the purification trap in the same supeconducting magnet, in the second homogeneous magnetic field region. A trap of a cylindrical type will be used, which should assure the measurement accuracy of $10^{-6}-10^{-7}$. It will enable mass measurements of many neutron-rich istopes not reachable anywhere else (e.g. of refractory elements) and will significantly broaden an experimental program at IGISOL.

In the third step of Penning trap development, nuclear spectroscopy in a Penning trap interior is foreseen. This means placing the detectors of a needed type directly inside the trap and positioning the radioactive sample in front of them. Such a scheme has following advantages over conventional spectroscopy:

- radioactive sample (ion cloud) constitutes a very thin, small size radioactive source with no backing, free of intensity attenuation, energy degradation and backscattering problems,

- due to a proper detector placement one can substantially decrease, in a given detector, background contribution from other types of radiation, e.g. gamma background in the electron spectrum. The latter can be achieved by placing the electron detector relatively far away (inside the trap) from the source, minimizing the solid angle for gammas while having still very high efficiency (solid angle of 50\%) for detecting electrons which are guided by the magnetic field. In particular, this would be beneficial for the conversion electron and beta spectroscopy,

- efficient passive shielding for a background radiation with the magnet cryostat.

It is planned to install and test the purification trap in the year 2000. A part of the necessary vacuum components (e.g. turbopumps) has been delivered already. This Penning trap system is developed in a close collaboration with GSI Darmstadt, where a similar trap project SHIPTRAP aiming at experiments with transuranium isotopes [6] is in preparation.

The ion trap development at IGISOL is done in a collaboration with other nuclear physics laboratories grouped in a european network EXOTRAPS (JYFL is a coordinator of this network). Within this collaboration, IGISOL group has participated (November 1999) in a precise nuclear mass measurement with the ISOLTRAP Penning trap set-up at ISOLDE, CERN [7]. The main result was the measurement, with an accuracy of $10^{-7}$, of the mass of ${ }^{33} \mathrm{Ar}$ with $\mathrm{T}_{1 / 2}=174$ ms (see [8]). This is the shortest half-life ever reached in the nuclear mass measurement with a Penning trap. It was possible due to the installation of 
an RFQ cooler/buncher at the ISOLTRAP apparatus. Thus, the nuclei with half-lives of the order of $0.1 \mathrm{~s}$ should also be available in the future for precise nuclear mass measurements at IGISOL.

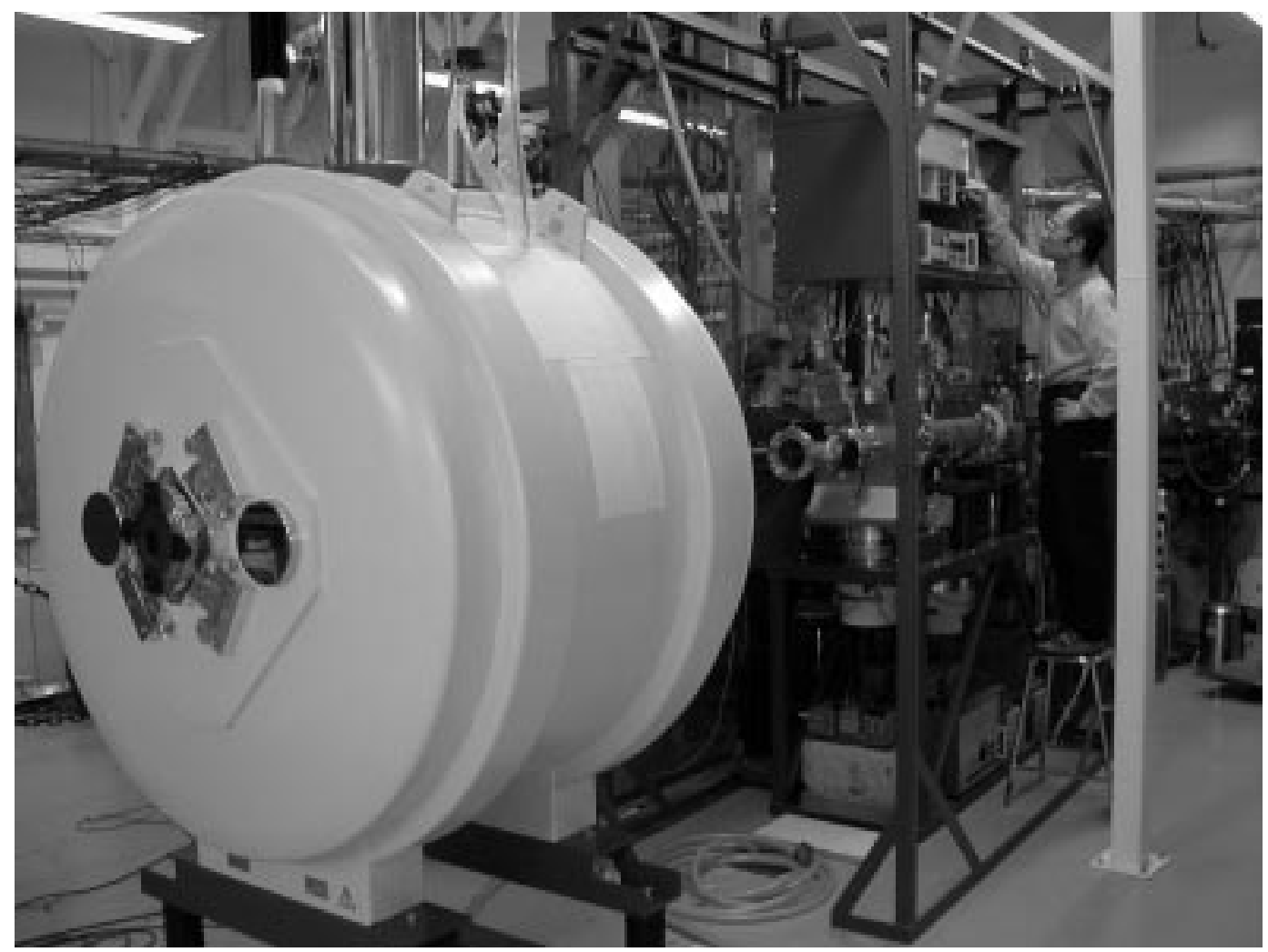

Fig. 1. The $7 \mathrm{~T}$ superconducting solenoid.

\section{References}

[1] A. Jokinen et al., JYFL Annual Report 1997, 15

[2] A. Nieminen et al., JYFL Annual Report 1998, 16

[3] A. Jokinen et al., contribution to this conference

[4] H. Raimbault-Hartmann et al., Nucl. Instr. Meth. B126 (1997) 378

[5] G. Savard et al., Phys. Lett. A158 (1991) 247

[6] J. Schoenfelder et al., contribution to this conference

[7] G. Bollen et al., Nucl. Instr. Meth. A368 (1996) 675

[8] F. Herfurth et al., contribution to this conference 